\title{
Effects of ketanserin on experimental colitis in mice and macrophage function
}

\author{
JUNHUA XIAO $^{1}$, LIMEI SHAO ${ }^{1}$, JIAQING SHEN ${ }^{2}$, WEILIANG JIANG ${ }^{3}$, \\ YUN FENG $^{3}$, PING ZHENG ${ }^{1}$ and FEI LIU ${ }^{1}$ \\ ${ }^{1}$ Department of Gastroenterology, Shanghai East Hospital, Tongji University, Shanghai 200092; \\ ${ }^{2}$ Department of Gastroenterology, The First Affiliated Hospital of Soochow University, Suzhou, Jiangsu 215006; \\ ${ }^{3}$ Department of Gastroenterology, Shanghai First People's Hospital, Shanghai Jiaotong University, Shanghai 200060, P.R. China
}

Received November 10, 2015; Accepted February 2, 2016

DOI: $10.3892 / \mathrm{ijmm} .2016 .2486$

\begin{abstract}
Ketanserin is a selective 5-hydroxytryptamine (serotonin)-2A receptor $\left(5-\mathrm{HT}_{2 \mathrm{~A}} \mathrm{R}\right)$ antagonist. Studies have suggested that ketanserin exerts anti-inflammatory effects independent of the baroreflex; however, the mechanisms involved remain unclear. Thus, in the present study, we aimed to evaluate the effects of ketanserin in colitis and the possible underlying mechanisms. The expression of $5-\mathrm{HT}_{2 \mathrm{~A}} \mathrm{R}$ was assessed in the colon tissues of patients with inflammatory bowel disease (IBD) and in mice with dextran sodium sulfate (DSS)-induced colitis. The therapeutic potential of ketanserin was investigated in the mice with colitis. In the colon tissue samples from the patients with IBD, a high expression level of $5-\mathrm{HT}_{2 \mathrm{~A}} \mathrm{R}$ was observed. Treatment with ketanserin attenuated the progression of experimental colitis in the mice, as indicated by body weight assessment, colon length, histological scores and cytokine release. The colonic macrophages from the ketanserin-treated mice with colitis exhibited a decreased production of inflammatory cytokines, with M2 polarization and impaired migration. The knockdown of 5- $\mathrm{HT}_{2 \mathrm{~A}} \mathrm{R}$ using siRNA partly abolished the inhibitory effects of ketanserin on the release of pro-inflammatory cytokines in bone marrow derived-macrophages (BMDMs), thus demonstrating that the inhibitory effects of ketanserin on the production of inflammatory cytokines are partly dependent on $5-\mathrm{HT}_{2 \mathrm{~A}} \mathrm{R}$. Ketanserin also inhibited the activation of nuclear factor- $\mathrm{KB}(\mathrm{NF}-\mathrm{\kappa} B)$ in BMDMs. In conclusion, the findings of the present study demonstrate that ketanserin alleviates colitis. Its anti-inflammatory effects may be due to the promotion
\end{abstract}

Correspondence to: Dr Fei Liu, Department of Gastrenterology, Shanghai East Hospital, Tongji University, 150 Jimo Road, Shanghai 200092, P.R. China

E-mail: doctorliufei@126.com

Key words: ketanserin, 5-hydroxytryptamine-2A receptor, macrophage, colitis of the anti-inflammatory function of macrophages through $5-\mathrm{HT}_{2 \mathrm{~A}} \mathrm{R} / \mathrm{NF}-\mathrm{\kappa B}$.

\section{Introduction}

Inflammatory bowel disease (IBD) is a group of chronic inflammatory conditions that affect the gastrointestinal tract and includes two main clinical forms: Crohn's disease (CD) and ulcerative colitis (UC). Although the precise etiology of IBD is not yet clearly understood, it has been suggested that an abnormal immune response against intestinal microbes plays a role in the pathogenesis of colitis (1).

5-Hydroxytryptamine (serotonin; 5-HT) modulates intestinal inflammation in the pathogenesis of colitis. Changes in the levels of 5-HT have been observed in experimental models of colitis and in patients with IBD (2-4). The knockdown 5-HT expression in tryptophan hydroxylase 1-deficient (TPH1 ${ }^{-/}$) mice, has been shown to lead to a delayed onset and to reduce the severity of dextran sodium sulfate (DSS)-induced colitis (5). Mucosal changes in IBD are characterized by ulcerative lesions with the prominent infiltration of immune cells and alterations in serotonin-producing enterochromaffin cells (6). 5-HT promotes the production of pro-inflammatory cytokines from macrophages (7), and nuclear factor- $\kappa \mathrm{B}$ (NF- $\mathrm{KB}$ ) signaling plays a critical role in the 5-HT-mediated activation of immune cells (8). However, the precise functions of 5-HT in intestinal inflammation are not yet fully understood. This is partly due to the fact that a wide variety of 5-HT receptors are expressed in the gut; $5-\mathrm{HT}_{1 \mathrm{~A}}, 5-\mathrm{HT}_{2 \mathrm{~A}}, 5-\mathrm{HT}_{2 \mathrm{~B}}, 5-\mathrm{HT}_{3}, 5-\mathrm{HT}_{4}$ and $5-\mathrm{HT}_{7}$ receptors are expressed in the intestines and some are known to have varying, and even contrasting roles.

Ketanserin is a selective 5- $\mathrm{HT}_{2 \mathrm{~A}}$ receptor $\left(5-\mathrm{HT}_{2 \mathrm{~A}} \mathrm{R}\right)$ antagonist with minor activity on the $\alpha 1$ adrenergic receptor (9). Previous studies have suggested that ketanserin exerts antiinflammatory effects independent of the baroreflex $(10,11)$. Ketanserin has been shown to inhibit the expression of inducible nitric oxide (NO) synthase (iNOS), NO, tumor necrosis factor- $\alpha$ (TNF- $\alpha$ ), and interleukin (IL)- 6 in lipopolysaccharide (LPS)-stimulated RAW264.7 cells (12). Moreover, the administration of ketanserin has been shown to decrease the expression of iNOS following endotoxic shock in mice (13). iNOS is primarily produced by macrophages. Macrophages 
participate in the immune response to the microenvironment in a polarized manner (14). Macrophages are often divided into 2 functional groups: ‘inflammatory' M1 macrophages (high IL-12 and iNOS expression) and 'wound-healing' M2 macrophages (low IL-12 expression, and high IL-10 expression) (15). M1 macrophages mainly produce inflammatory cytokines (TNF- $\alpha$ and IL-12) and iNOS. The production of these cytokines promotes naïve $\mathrm{CD} 4^{+} \mathrm{T}$ cells to differentiate into $\mathrm{Th} 1$ and Th17 cells (16). M2 macrophages produce the anti-inflammatory cytokine, IL-10, and are efficient at recruiting regulatory $\mathrm{T}$ cells that promote tolerance to local immune responses (16). In this study, we examined the hypothesis that ketanserin may attenuate colitis by modulating the function of macrophages, thus alleviating colitis.

\section{Materials and methods}

Human tissue samples. Intestinal specimens were obtained from 15 patients with IBD. Normal tissue specimens were obtained from patients receiving colonoscopy for colorectal cancer screening. The tissues were frozen in liquid nitrogen and stored at $-80^{\circ} \mathrm{C}$ for reverse transcription-quantitative PCR (RT-qPCR) analyses, or were embedded in optimum cutting temperature (OCT) compound for immunofluorescence staining. All studies involving samples obtained from human participants were approved by the Ethics Committee of the Humanitas Clinical and Research Center of Shanghai East Hospital, Shanghai, China. Written informed consent was obtained from each patient or their family before initiating the study protocol.

Mice. A total of 30 C57BL/6 male mice (8 to 10 weeks old, weighing $20 \mathrm{~g}$ ) were purchased from Slaccas Laboratory Animal Co., Ltd. (Shanghai, China) and all experiments were performed under specific pathogen-free conditions at the Animal Resources facilities of Shanghai East Hospital. All animal experiments were performed following approval by the Animal Care and Use Committee of Shanghai East Hospital.

Induction of colitis by DSS. The mice were randomly divided into 3 groups as follows: the control group, DSS treatment group and the DSS plus ketanserin treatment group $(n=10$ in each group). The mice were administered 4\% (wt/vol) DSS (MP Biomedicals, Solon, OH, USA) dissolved in their drinking water for 7 days to induce acute experimental colitis. Ketanserin (10 mg/kg; Janssen Pharmaceuticals, Beijing, China) or phosphate-buffered saline (PBS; vehicle control; administered to the mice in the DSS treatment group) were administered intraperitoneally once daily for 3 days when the administration of DSS began. The weight of the mice was recorded daily using an electronic weighing system (Practum 612-1CN; Sartorius AG, Goettingen, Germany). On day 7 , the mice were sacrificed by an intraperitoneal injection of $4 \%$ sodium pentobarbital and the colons were obtained for the measurement of colon length. For histological analysis, the colons were excised and fixed in $4 \%$ paraformaldehyde. Subsequentoy, $1 \mathrm{~cm}$ of the distal colon of each mouse was paraffin-embedded. The paraffin-embedded sections were cut (4- $\mu \mathrm{M}$-thick) using a microtome and stained with hematoxylin and eosin. For gene and protein expression analyses, the colons were immediately frozen in liquid $\mathrm{N}_{2}$ and stored at $-80^{\circ} \mathrm{C}$.
$R T-q P C R$. Total RNA was extracted from the colon tissues or lamina propria macrophages (LP-macrophages) and bone marrow derived-macrophages (BMDMs) using TRIzol reagent (Invitrogen, Carlsbad,CA, USA) according to the manufacturer's instructions. Complementary DNA (cDNA) was synthesized using the PrimeScript ${ }^{\mathrm{TM}}$ RT reagent kit (Takara, Shiga, Japan). Messenger RNA (mRNA) transcripts were analyzed by quantitative PCR using SYBR ${ }^{\circledR}$ Premix Ex Taq ${ }^{\mathrm{TM}}$ (Takara) with an Applied Biosystems StepOne/StepOnePlus Real-Time PCR System. Gene expression was normalized to the expression of glyceraldehyde 3-phosphate dehydrogenase (GAPDH) and the relative expression levels were quantified using the $2^{-\Delta \Delta \mathrm{CT}}$ method. The primer pairs used are listed in Table I.

Western blot analysis. Colon proteins were extracted using RIPA buffer supplemented with protease inhibitors (Shanghai Shenggong Co., Ltd., Shanghai, China). A total of $40 \mu$ g protein was separated by $10 \%$ sodium dodecyl sulfate-polyacrylamide gel electrophoresis (SDS-PAGE), and the gels were then electrotransferred onto nitrocellulose filter membranes (NC; Whatman, Alameda, CA, USA). The membranes were incubated with antibodies against total NF- $\kappa \mathrm{B}(\mathrm{p} 65$; Cat. no. 8424; 1:1,000), phosphorylated (p-)NF-кB (p65; Cat. no. 3033; 1:1,000), or GAPDH (Cat. no. 8884; 1:1,000) (all from Cell Signaling Technology, Beverly, MA, USA) overnight at $4^{\circ} \mathrm{C}$. The membranes were then incubated with an IRDye 800CW-conjugated secondary antibody (Rockland, Hamburg, Germany) for $1 \mathrm{~h}$ at room temperature. Images were acquired using an Odyssey infrared imaging system (LI-COR Biosciences, Inc., Lincoln, NE, USA).

Flow cytometric analysis. Briefly, the colons were longitudinally cut and washed in PBS containing $1 \%$ fetal bovine serum and $1 \mathrm{mMDTT}$ for $10 \mathrm{~min}$ at room temperature 3 times. Subsequently, the colon tissues were digested in complete RPMI-1640 medium (Invitrogen) supplemented with $0.5 \mathrm{mg} / \mathrm{ml}$ collagenase type VIII (Sigma-Aldrich, St. Louis, MO, USA), $20 \mathrm{mg} / \mathrm{ml}$ DNase I (Roche Diagnostics, Mannheim, Germany) for $60 \mathrm{~min}$ at $37^{\circ} \mathrm{C}$ in a shaking water bath. The cell suspension was passed through a $100-70 \mu \mathrm{m}$ filter and then resuspended in $1.077 \mathrm{~g} / \mathrm{cm}^{3}$ iso-osmotic metrizamide medium (Accurate Chemical \& Scientific Corp., Westbury, NY, USA). Following centrifugation at 1,500 x g for $15 \mathrm{~min}$ at room temperature, the low-density fraction was collected. For FACS analysis of the colon samples, the cells were stained with antibodies to CD45 (17-0451), CD11c (12-0114), MHC-II (56-5321), F4/80 (15-4801), CD11b (11-0112), Ly-6G (Gr-1; 53-5931) (all from eBioscience, San Diego, CA, USA) and CD206 (141711) (from BioLegend, San Diego, CA, USA). The influx of neutrophils, dendritic cells (DCs) and macrophages was determined by the frequency of macrophages, neutrophils and DCs in CD45 cells in the LP of colons.

Isolation and stimulation of murine macrophages. To isolate the LP-macrophages from the lamina propria of the mouse colons, the colons were washed with PBS and cut into small sections. The latter were then incubated with $5 \mathrm{mM}$ EDTA and $3 \% \mathrm{FCS}$ in $\mathrm{Ca}^{2+}$ - and $\mathrm{Mg}^{2+}$-free Hanks balanced salt solution for $30 \mathrm{~min}$ at $37^{\circ} \mathrm{C}$ with stirring. The small sections were collected and were then digested with RPMI-1640 containing 5\% FCS, $1 \mathrm{mg} / \mathrm{ml}$ collagenase type IV and $0.1 \mathrm{mg} / \mathrm{ml}$ DNase for $1 \mathrm{~h}$ at 
Table I. Sequences of primers used for RT-qPCR.

\begin{tabular}{|c|c|c|}
\hline Gene product & Forward primer & Reverse primer \\
\hline $\operatorname{mTNF}-\alpha$ & 5'-gtcgtagcaaaccaccaagtg-3' & 5'-cagatttgtgttggtccttc-3' \\
\hline mIL-1 $\beta$ & 5'-atctcgcagcagcacatcaac-3' & 5'-cacgggaaagacacaggtagc-3' \\
\hline mIL-6 & 5'-acaagtcggaggettaattacacat-3' & 5'-ttgccattgcacaactcttttc-3' \\
\hline mIL-10 & 5'-gctcttactgactggcatgag-3' & 5'-cgcagctctaggagcatgtg-3' \\
\hline mIL-12 p40 & 5'-agacatggagtcataggctctg-3' & $5^{\prime}$-ccattttcettcttgtggagca-3' \\
\hline miNOS & 5'-gccaccaacaatggcaaca-3' & $5^{\prime}$-cgtaccggatgagctgtgaatt-3' \\
\hline $\mathrm{mKC}$ & 5'-cttgaaggtgttgecctcag-3' & 5'-tggggacaccttttagcatc-3" \\
\hline mCXCL12 & 5'-aaaccagtcagcctgagctac-3' & $5^{\prime}$-aagggcacagtttggagtgtt-3' \\
\hline mCD206 & 5'-ttcggtggactgtggacgagca-3' & 5'-ataagccacctgccactccggt-3' \\
\hline mCD32 & 5'-aatcctgccgttcctactgatc-3' & 5 '-gtgtcaccgtgtcttccttgag-3' \\
\hline $\mathrm{m} 5-\mathrm{HT}_{2 \mathrm{~A}^{-}}$ & $5^{\prime}$-cgactcgaggctctaccta- $3^{\prime}$ & $5^{\prime}$-ttcgcaggagaggttggttc- $3^{\prime}$ \\
\hline $\mathrm{h} 5-\mathrm{HT}_{2 \mathrm{~A}} \mathrm{R}$ & 5 '-tgaagtcagcaaaacagaaacca-3' & 5'-gactcgctgcatctctcaca-3' \\
\hline
\end{tabular}

TNF- $\alpha$, tumor necrosis factor- $\alpha$; IL-1 $\beta$, interleukin- $1 \beta$; iNOS, inducible nitric oxide synthase; KC, keratinocyte-derived chemokine; 5- $\mathrm{HT}_{2 \mathrm{~A}}-\mathrm{R}, 5$-hydroxytryptamine-2A receptor.

$37^{\circ} \mathrm{C}$. The liberated cells were collected through a stainless steel sieve. Following centrifugation $\left(500 \mathrm{x} \mathrm{g}, 4^{\circ} \mathrm{C}\right)$ and washing with PBS 3 times, the intestinal macrophages were enriched by positive selection with anti-CD11b Dynabeads following the manufacturer's instructions (Miltenyi Biotec $\mathrm{GmbH}$, Bergisch Gladbach, Germany). The cells obtained were tested for the expression of macrophage markers by flow cytometry $\left(\mathrm{CD} 45^{+} \mathrm{CD} 11 \mathrm{~b}^{+} \mathrm{CD} 11 \mathrm{c}^{-} \mathrm{F} 4 / 80^{+}\right)$; cells with a purity $>85 \%$ were used in the experiments.

LP-macrophages $\left(5 \times 10^{4}\right)$ were cultured in complete RPMI-1640 medium and treated with $100 \mathrm{ng} / \mathrm{ml}$ LPS for $6 \mathrm{~h}$. Supernatants were collected for the measurement of cytokine levels and the attached cells were lysed for RNA extraction. Briefly, the levels of the cytokines, IL-1 $\beta$, IL-6, IL-10 and $\mathrm{TNF}-\alpha$, were evaluated in the supernatant from the macrophage cultures by enzyme-linked immunosorbent assay (ELISA), following the manufacturer's instructions (R\&D Systems, Inc., Minneapolis, MN, USA).

Migration and phagocytosis of LP-macrophages. The migration of the LP-macrophages induced by C-X-C motif chemokine 12 [CXCL12, also known as stromal cell-derived factor 1 (SDF-1)] was assessed using a 48-well microchemotaxis chamber (Neuro Probe, Gaithersburg, MD, USA). CXCL12 (50 nM; 250-20B, PeproTech, Rocky Hill, NJ, USA) was placed in wells $(30 \mu \mathrm{l})$ of the lower compartment, and LP-macrophages ( $50 \mu \mathrm{l}$ of a $5 \times 10^{* 4} / \mathrm{ml}$ suspension) were seeded in the wells of the upper compartment. The chamber was incubated in a humidified environment at $37^{\circ} \mathrm{C}$ with $5 \% \mathrm{CO}_{2}$ for $4 \mathrm{~h}$. The membrane was removed, fixed in methanol, and stained with crystal violet solution for 30 min followed by destaining with water. The cells that had migrated across the membrane were counted using a microscope (Leica TCS SP8 CARS confocal microscope, Leica Microsystems GmbH, Wetzlar, Germany). The chemotaxis index was calculated as the ratio of the number of cells that had migrated towards the chemoattractant divided by the number of cells that had migrated towards the medium.
The LP-macrophages $\left(5 \times 10^{* 4}\right)$ were infected with $E$. coli at an MOI of 1:10 for $1 \mathrm{~h}$ in complete RPMI-1640 medium without antibiotics, and then incubated in complete medium containing $40 \mu \mathrm{g} / \mathrm{ml}$ gentamycin for $120 \mathrm{~min}$. The cells were then lysed and seeded on LB agar plates. Following overnight incubation at $37^{\circ} \mathrm{C}$, bacterial colonies (CFUs) were counted as a measure of intracellular bacteria.

Immunofluorescence staining. For the human tissues, $4-\mu \mathrm{m}$-thick frozen sections of intestinal specimens were fixed in cold acetone for $10 \mathrm{~min}$ at $-20^{\circ} \mathrm{C}$ and blocked with $5 \%$ BSA for $1 \mathrm{~h}$ at room temperature, then incubated with a primary rabbit anti-human $5-\mathrm{HT}_{2 \mathrm{~A}} \mathrm{R}$ antibody (ab66049; rabbit polyclonal to $5-\mathrm{HT}_{2 \mathrm{~A}} \mathrm{R}$; reacts to mouse, rat, human; Abcam, Cambridge, UK; 1:500) overnight at $4^{\circ} \mathrm{C}$. Mouse anti-human CD68 antibody (MCA5709; mouse anti-human CD68, monoclonal antibody; AbD Serotec, Kidlington, UK; 1:500) (overnight at $4^{\circ} \mathrm{C}$ ) was subsequently used to detect the macrophages. For the murine colon tissues, $5-\mathrm{HT}_{2 \mathrm{~A}} \mathrm{R}$ expression was detected by overnight incubation at $4{ }^{\circ} \mathrm{C}$ with rabbit anti-mouse 5- $\mathrm{HT}_{2 \mathrm{~A}} \mathrm{R}$ antibody (ab66049; rabbit polyclonal to $5-\mathrm{HT}_{2 \mathrm{~A}} \mathrm{R}$; reacts to mouse, rat, human; Abcam; 1:300). Subsquently, rat anti-mouse CD68 antibody (MCA1957GA; rat anti-mouse CD68, monoclonal antibody; AbD Serotec; 1:500) was used overnight at $4^{\circ} \mathrm{C}$. For both analyses, AlexaFluor 488- and Alexa-Fluor 555-conjugated antibodies [goat anti-rabbit IgG (ab150077, goat anti-rat IgG (ab150158) and goat anti-mouse IgG (ab150118); all from Abcam, Cambridge, $\mathrm{UK} ; 1: 1,000 ; 1 \mathrm{~h}$ at room temperature] were used as secondary antibodies, followed by incubation with $1 \mu \mathrm{g} / \mathrm{ml}$ DAPI ( $20 \mathrm{~min}$, room temperature). The sections were finally visualized under a confocal microscope (Olympus, Tokyo, Japan). Images were captured using FluoView software.

Transfection with small interfering RNA (siRNA). siRNA targeting the $5-\mathrm{HT}_{2 \mathrm{~A}} \mathrm{R}$ (Genepharm Biotech, Shanghai, China) was synthesized (GACAACUGUCGUGAUUAUUTT) and control siRNA (UUCUCCGAACGUGUCACGUTT) was also 
A
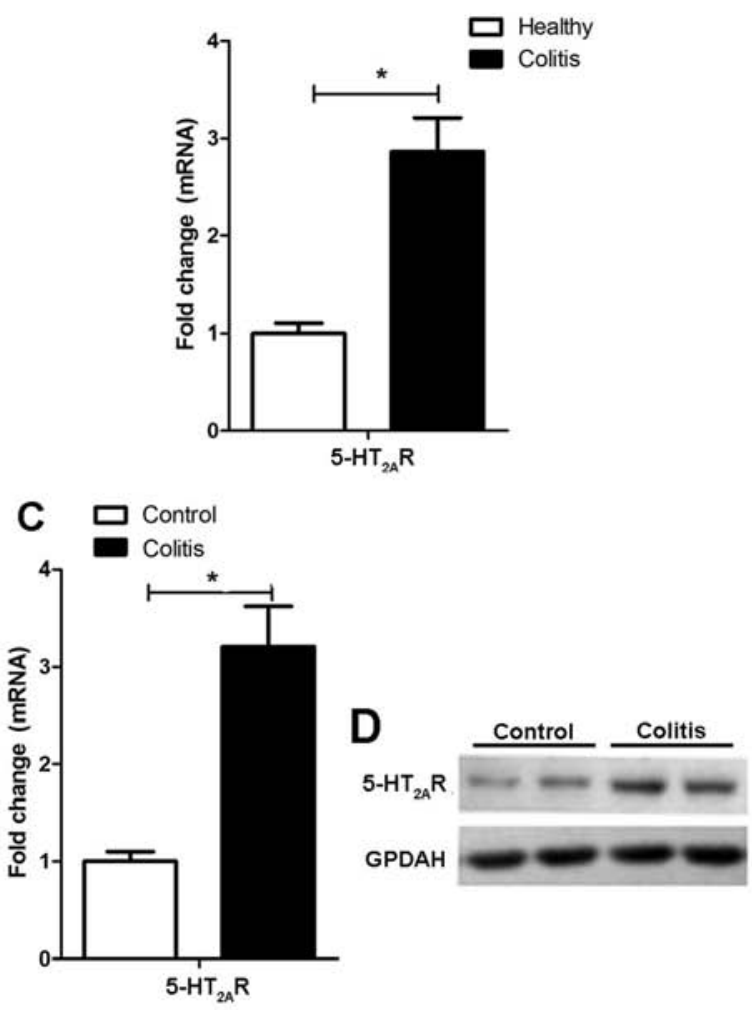

B
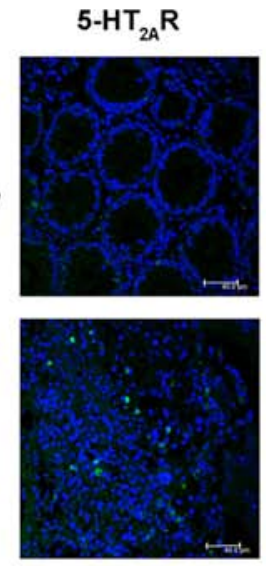

E

Control
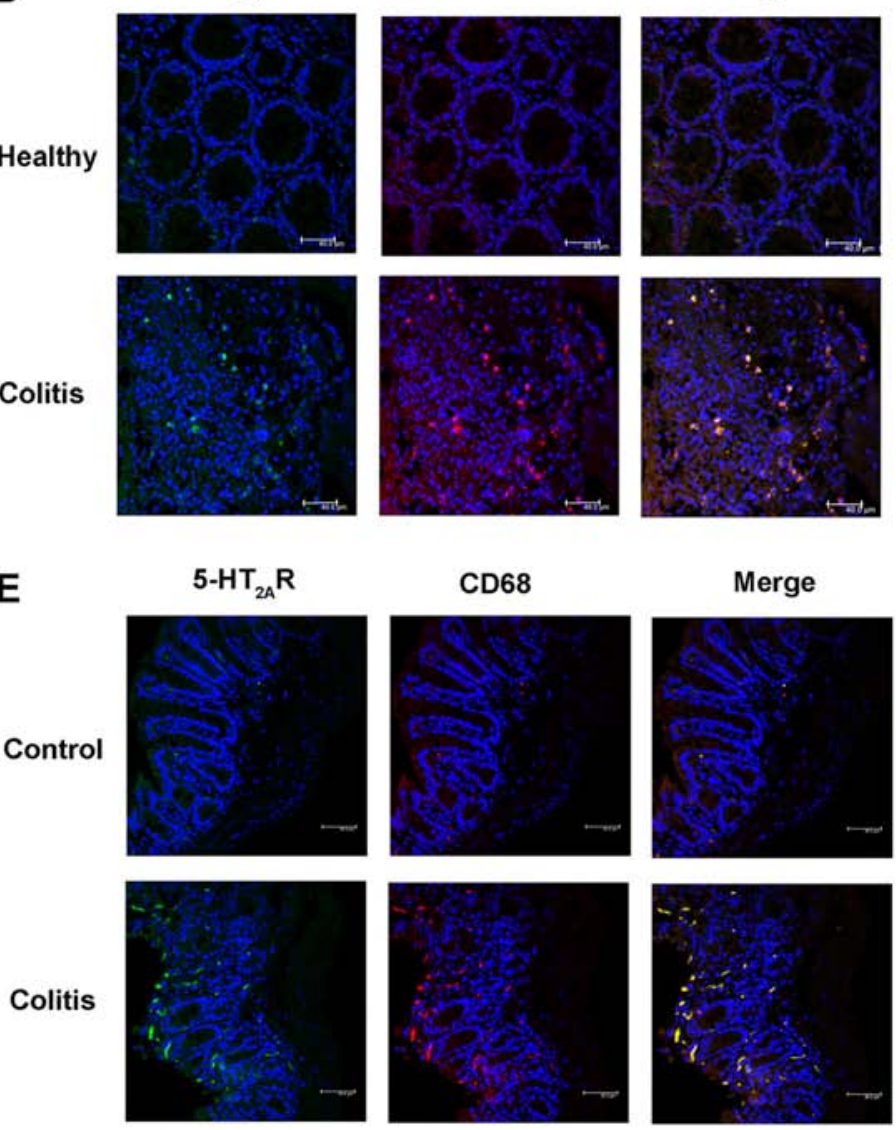

Figure 1.5-Hydroxytryptamine-2A receptor $\left(5-\mathrm{HT}_{2 \mathrm{~A}} \mathrm{R}\right)$ expression is upregulated in the colons of patients with inflammatory bowel disease (IBD) and in mice with dextran sodium sulfate (DSS)-induced colitis, and is specifically enhanced in macrophages. (A) Relative mRNA expression of 5-HT ${ }_{2 \mathrm{~A}} \mathrm{R}$ in healthy subjects and patients wtih IBD estimated by RT-qPCR. (B) Representative images of immunofluorescence staining of colonic mucosa samples of healthy subjects and in patients with IBD showing 5- $\mathrm{HT}_{2 \mathrm{~A}} \mathrm{R}^{+}$cells (in green) and $\mathrm{CD} 68^{+}$cells (in red). Co-localization of 5- $\mathrm{HT}_{2 \mathrm{~A}} \mathrm{R}$ with the macrophage marker, $\mathrm{CD} 68^{+}$, is shown in the merged images. 5- $\mathrm{HT}_{2 \mathrm{~A}} \mathrm{R}$ expression in mice with DSS-induced colitis estimated by (C) RT-qPCR and (D) western blot analysis. Representative images of immunofluorescence staining of 5- $\mathrm{HT}_{2 \mathrm{~A}} \mathrm{R}^{+}$cells (in green) and $\mathrm{CD} 68^{+}$cells (in red) in the colonic mucosa samples of mice treated with or without DSS. (E) Co-localization of 5- $\mathrm{HT}_{2 \mathrm{~A}} \mathrm{R}$ with $\mathrm{CD} 68$ is shown in the merged images.

used. BMDMs were isolated from bone marrow cells obtained from the mice. Three C57BL/6 male mice (8 to 10 weeks old, weighing $20 \mathrm{~g}$ ) were sacrificed as described for the mice in the other experiments, and bone marrow cells were isolated from femurs and cultured with RPMI-1640 supplemented with $10 \% \mathrm{FBS}, 1 \%$ penicillin and streptomycin (Invitrogen) and $10 \%$ L929 conditioned medium. The culture fluid was exchanged for fresh culture medium every 4 days. Under these conditions, adherent macrophage monolayers were obtained within 8-10 days. The BMDMs were then transfected with the siRNAs as previously described (17). Following transfection, the BMDMs $\left(1 \times 10^{6} / \mathrm{ml}\right)$ were pre-incubated with ketanserin $(10 \mu \mathrm{M})$ for $10 \mathrm{~min}$ and then stimulated with LPS (100 ng/ml) and 5-HT (100 nmol/l) for $12 \mathrm{~h}$. RNA was then extracted for PCR.

Statistical analysis. The statistical significance of the differences between the treatment and control groups was determined using a Student's t-test. Data were analyzed with one-way analysis of variance (ANOVA), followed by the Student's t-test for experiments involving only 2 groups, and Dunnett's t-test for experiments involving $>2$ groups. All data are expressed as the means \pm standard deviation (SD). Statistical significance was set at $\mathrm{P}<0.05$.

\section{Results}

5-HT ${ }_{2 A} R$ expression is elevated in both patients with IBD and in mice with experimental colitis, and is specifically enhanced in macrophages. We first examined the expression of $5-\mathrm{HT}_{2 \mathrm{~A}} \mathrm{R}$ in patients with IBD and in mice with DSS-induced experimental colitis. The $5-\mathrm{HT}_{2 \mathrm{~A}} \mathrm{R}$ mRNA level was increased in the patients with IBD (Fig. 1A) compared to the normal tissue specimens, and $5-\mathrm{HT}_{2 \mathrm{~A}} \mathrm{R}$ protein expression was also elevated in the patients with IBD, as detected by immunofluorescence staining (Fig. 1B). $5-\mathrm{HT}_{2 \mathrm{~A}} \mathrm{R}$ is known to be expressed on the surface of lymphocytes, natural killer (NK) cells and monocytes/macrophages/DCs $(18,19)$. We observed a specific co-localization of $5-\mathrm{HT}_{2 \mathrm{~A}} \mathrm{R}$ with the CD68 macrophage marker (Fig. 1B), reflecting that $5-\mathrm{HT}_{2 \mathrm{~A}} \mathrm{R}$ expression is enhanced in macrophages. The $5-\mathrm{HT}_{2 \mathrm{~A}} \mathrm{R}$ expression level was also upregulated in the mice with DSS-induced experimental colitis (Fig. 1C and D) and was co-localized with CD68 postivity (Fig. 1E). These data suggest that the synthesis of 
A

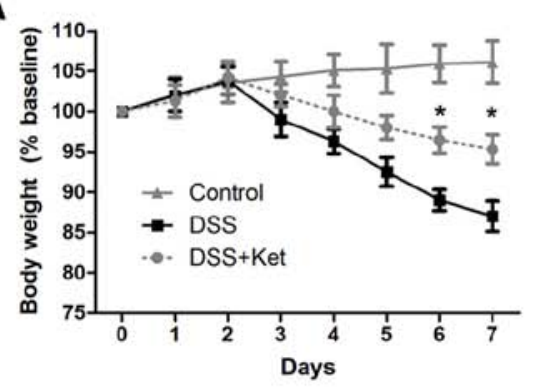

D

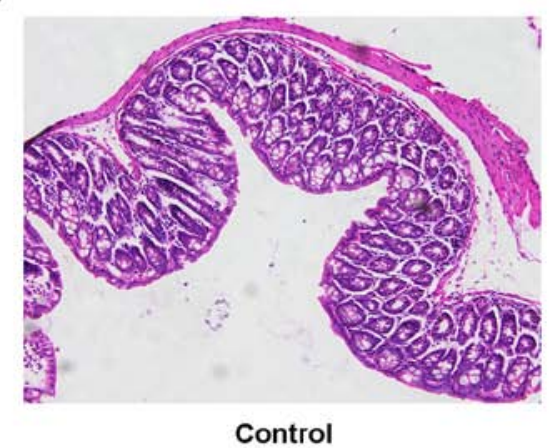

B
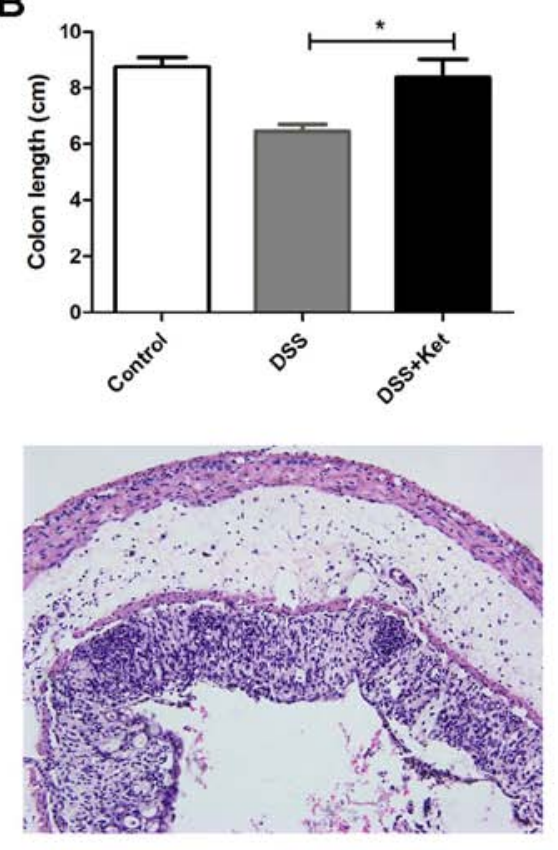

DSS
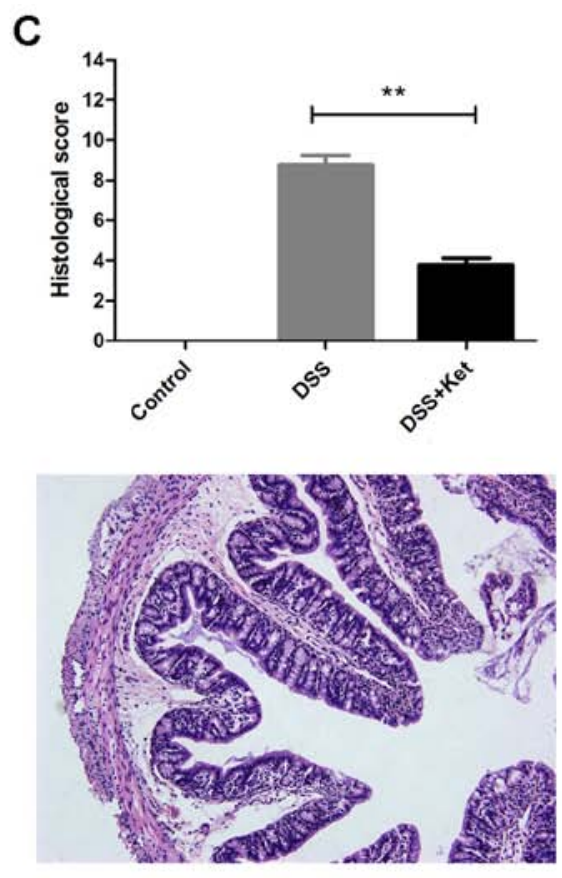

DSS+Ket

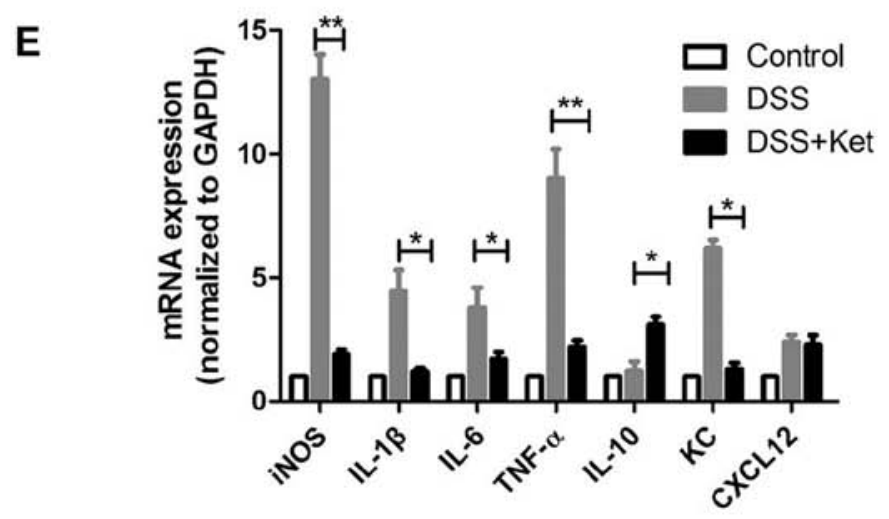

Figure 2. Ketanserin suppresses inflammation of the colon in mice with dextran sodium sulfate (DSS)-induced acute experimental colitis. Mice received DSS with or without ketanserin $(10 \mathrm{mg} / \mathrm{kg}$, intraperitoneally, 3 days). (A) Loss of body weight was evaluated and expressed as a percentage of the initial weight. (B) Colon length was measured. (C) Colitis histological score. (D) Representative images of hematoxylin and eosin (H\&E)-stained colon sections are shown (x200 magnification). (E) On day 7 following dextran sulfate sodium (DSS) administration, distal colon sections were harvest and the relative mRNA expression of inflammatory mediators was measured by RT-qPCR. Data are represented as the means \pm SD of 3 independent experiments. ${ }^{*}<0.05$ and ${ }^{* *} \mathrm{P}<0.01$. Ket, ketanserin.

$5-\mathrm{HT}_{2 \mathrm{~A}} \mathrm{R}$ is induced in the inflamed colon and that it is mostly expressed in macrophages.

The administration of ketanserin attenuates the development of DSS-induced acute experimental colitis in mice. To investigate whether ketanserin reduces susceptibility to colitis, we induced colitis in mice using DSS. The mice with DSS-induced colitis exhibited a continuous decrease in body weight from day 4 to day 7 and shortened colon lengths. By contrast, the administration of ketanserin during the induction of colitis significantly prevented the decrease in body weight (Fig. 2A) and colon shortening (Fig. 2B). A histological examination of the colons of the mice with DSS-induced colitis revealed severe inflammation with ulcerative lesions, loss of crypts and the infiltration of inflammatory cells, whereas treatment with ketanserin alleviated these histological changes and damage to the colon, characterized by a decrease in the loss of architecture, fewer ulcerative lesions, and a decrease in inflammatory cell infiltration into the inflamed mucosa (Fig. 2C and D). Our data therefore suggest that ketanserin exerts a potent therapeutic effect, ameliorating DSS-induced colitis.

DSS is directly toxic to the colonic epithelium and triggers inflammation in the gut with high levels of inflammatory mediators, such as iNOS, IL-1 $\beta$ and IL-6 $(20,21)$. We thus evaluated the effects of ketanserin on the production of inflammatory mediators in the colonic mucosa by RT-qPCR. Compared with the vehicle-treated mice with colitis (DSS group), the ketanserin-treated mice exhibited significantly lower mRNA levels of keratinocyte-derived chemokine ( $\mathrm{KC}$; a major chemoattractant for neutrophils), iNOS, TNF- $\alpha$, IL- $1 \beta$ and IL-6 (Fig. 2E). Moreover, the transcript levels of IL-10 were found to be higher in the ketanserin-treated mice. The levels 

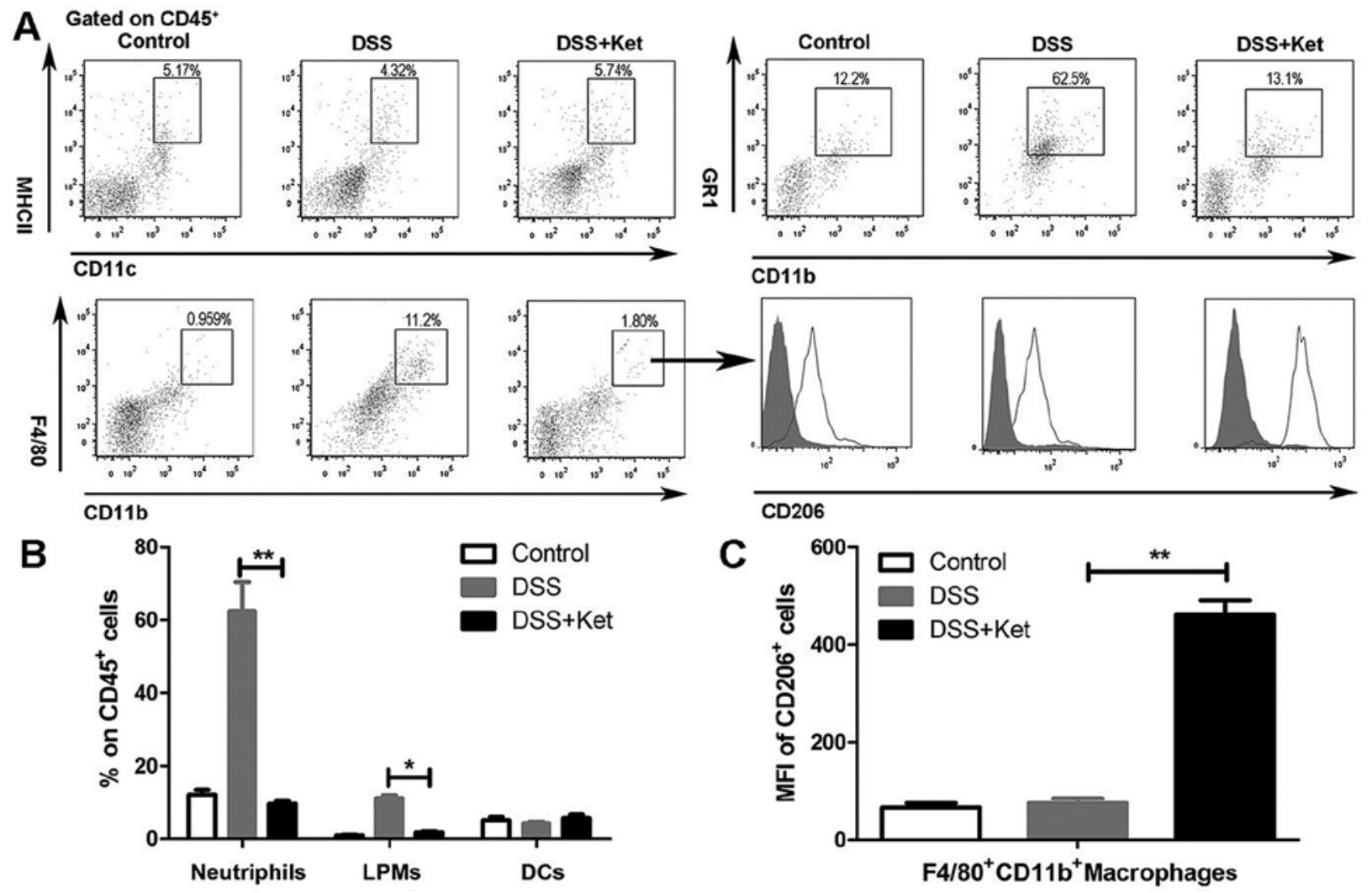

Figure 3. Effects of ketanserin on the recruitment of innate immune cells in the colons of mice with dextran sodium sulfate (DSS)-induced colitis. Seven days after the administration of DSS and treatment with or without ketanserin, (A) the frequencies of macrophages $\left(\mathrm{CD} 11 \mathrm{~b}^{+} \mathrm{F} 4 / 80^{+}\right)$, neutrophils $\left(\mathrm{CD} 11 \mathrm{~b}^{+} \mathrm{Gr}-\mathrm{1}^{+}\right)$, and dendritic cells $(\mathrm{DCs})\left(\mathrm{MHCII}^{+} \mathrm{CD} 11 \mathrm{c}^{+}\right)$in isolated colonic lamina propria (LP) cells from mice in the indicated groups were determined by flow cytometry. Representative plots are shown. The MFI of CD206 ${ }^{+}$cells was determined. (B) The frequency of macrophages, neutrophils and DCs in CD45 cells in the LP of colons. (C) The MFI of $\mathrm{CD} 206^{+}$cells in $\mathrm{F} 4 / 80^{+} \mathrm{CD} 11 \mathrm{~b}^{+}$macrophages. The results are shown as the means $\pm \mathrm{SD}$ from 5 individual mice per group. " $\mathrm{P}<0.05$ and ${ }^{* *} \mathrm{P}<0.01$. Ket, ketanserin; LPMs, lamina propria macrophages.

of CXCL12, the major chemoattractant for DCs, remained unaltered.

Ketanserin reduces the infiltration of neutrophils and macrophages into the intestinal mucosa in colitis. Innate immune cells are the major sources of inflammatory mediators in DSS-induced colitis $(22,23)$. We thus investigated whether the effects of ketanserin alter the infiltration of innate immune cells, thus influencing the inflammatory response in the colon. In the mice with DSS-induced colitis, the percentage of neutrophils, macrophages and DCs in the $\mathrm{CD} 45^{+}$cells was significantly increased in the colonic lamina propria on day 7 compared with the control mice (Fig. 3A). Consistently, treatment with ketanserin resulted in a decrease in the influx of neutrophils and macrophages into the inflamed colons of the mice (Fig. 3B). Minor differences in the relative percentage of DCs in the $\mathrm{CD}^{+} \mathrm{T}$ cell population were observed. We also found that the colonic macrophages in the ketanserintreated group expressed higher levels of CD206, a marker of M2 macrophages, compared to the vehicle-treated mice with colitis (DSS group; Fig. 3A and C).

Ketanserin inhibits the migration of LP-macrophages without affecting the ability of bacterial phagocytosis. As the ketanserin-treated mice exhibited a reduced influx of macrophages into the colonic lamina propria, we then determined whether ketanserin suppresses the chemotaxis of macro- phages. As CXCL12 is a critical regulator of macrophage migration $(24,25)$, the migration of LP-macrophages through an $8-\mu \mathrm{m}$ filter towards CXCL12 $(50 \mathrm{nM})$ placed in the lower chamber was examined. The chemotaxis index indicated that ketanserin significantly reduced the migration of macrophages towards CXCL12 (Fig. 4A and B).

It has been shown that 5-HT modulates the activity of the phagocytosis of bacteria by macrophages through $5-\mathrm{HT}$ receptors (26). Thus, to determine whether ketanserin affects the phagocytic ability of macrophages, LP-macrophages were infected with $E$. coli and viable intracellular bacteria CFUs were counted in order to evaluate bacterial phagocytosis. We found that the ability of the LP-macrophages to phagocytize E. coli in the ketanserin-treated mice with colitis did not differ from that in the vehicle-treated mice with colitis (DSS group; Fig. 4C).

Ketanserin inhibits the expression of pro-inflammatory mediators and influences the polarization of LP-macrophages. We then evaluated the release of cytokines in the supernatants of LP-macrophages obtained from the inflamed colon. In line with our hypothesis, the secretion of TNF- $\alpha$, IL-1 $\beta$ and IL-6 was significantly decreased in the ketanserin-treated mice (Fig. 5A-C). In addition, the ketanserin-treated mice produced higher amounts of IL-10 (Fig. 5D).

Since the LP-macrophages from the ketanserin-treated mice with colitis exhibited a high level of CD206, a marker of M2 macrophages (as shown by FACS analysis), we therefore 
A

Control

DSS

Ket+DSS

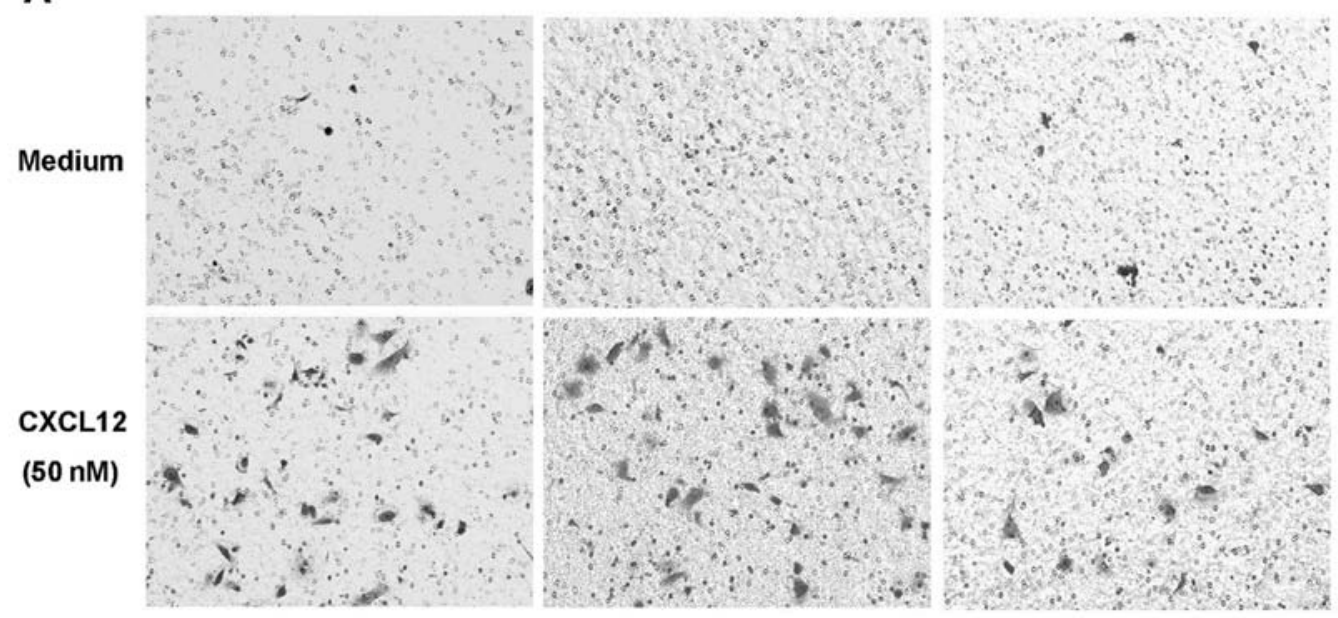

B

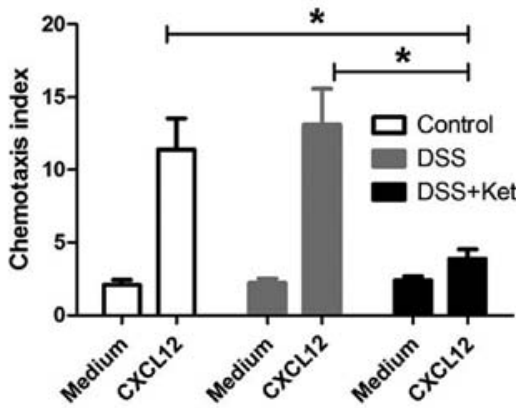

C

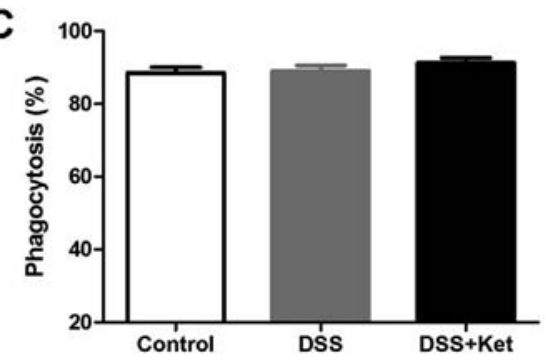

Figure 4. Ketanserin significantly inhibits LP-macrophage chemotaxis, but not phagocytosis. LP-macrophages were isolated from mice with dextran sodium sulfate (DSS)-induced colitis treated or not with ketanserin on day 7. Migration of LP-macrophages induced by CXCL12 was assessed using a microchemotaxis chamber. (A) Sample images of cells that had migrated through the polycarbonate filter following incubation for $4 \mathrm{~h}$ with medium (upper panel) and CXCL12 (lower panel). (B) Chemotaxis index was calculated as the ratio of the number of cells that tad migrated toward the stimuli over those that had migrated toward the medium. (C) Bacterial phagocytosis of LP-macrophages was evaluated by gentamycin protection assay following infection with $E$. coli at the indicated time points. The graphs depict the means \pm SD of 3 independent experiments. ${ }^{*} p<0.05$. Ket, ketanserin.

measured the mRNA levels of iNOS, CD32 and IL-12 p40, as M1 polarization markers, and the levels of CD206 and IL-10, as M2 polarization markers in the LP-macrophages in all 3 groups. Of note, the LP-macrophages from the ketanserintreated mice exhibited a notably decreased expression of iNOS, CD32 and IL-12 p40 (Fig. 5E), but elevated levels of CD206 and IL-10 (Fig. 5F). Taken together, these data indicate that ketanserin modulates LP-macrophage function by reducing the production of pro-inflammatory cytokines and promoting M2 as opposed to M1 polarization.

Ketanserin inhibits the expression of pro-inflammatory cytokines in BMDMs through $5-H T_{2 A} R / N F-\kappa B$. In order to determine whether $5-\mathrm{HT}_{2 \mathrm{~A}} \mathrm{R}$ plays a role in the effects of ketanserin, we used 5- $\mathrm{HT}_{2 \mathrm{~A}} \mathrm{R}$ siRNA to downregulate the expression of $5-\mathrm{HT}_{2 \mathrm{~A}} \mathrm{R}$ in BMDMs. The LPS/5HT-induced expression of the pro-inflammatory cytokines, IL-1 $\beta$ (Fig. 6A), TNF- $\alpha$ (Fig. 6B), IL-6 (Fig. 6C) and iNOS (Fig. 6D), was inhibited by treatment with ketanserin in the BMDMs. The knockdown of $5-\mathrm{HT}_{2 \mathrm{~A}} \mathrm{R}$ by siRNA partly abolished the inhibitory effects of ketanserin on the expression of these pro-inflammatory cytokines in the BMDMs stimulated with LPS plus 5-HT (Fig. 6). In a previous study, it was suggested that the anti-inflammatory effects of ketanserin are partly dependent on the arterial baroreflex (13). In the present study, we found that ketanserin decreased the levels of inflammatory cytokines in BMDMs, suggesting that the anti-inflammatory effects of ketanserin are not entirely dependent on the baroreflex. Moreover, these effects were dimished by transfection with siRNA targeting 5-HT2AR, thus demonstrating that the inhibitory effects of ketanserin are partly-dependent on 5-HT2AR.

$\mathrm{NF}-\kappa \mathrm{B}$ is a critical transcription factor for the inflammatory response (27). It functions as a pro-inflammatory factor and participates in the pathophysiology of IBD (28). Thus, in order to elucidate the mechanisms responsible for the antiinflammatory effects of ketanserin, we examined its effects on the activation of the NF- $\kappa \mathrm{B}$ pathway in BMDMS. Changes in the levels of p-NF- $\mathrm{B}$ p65 in the BMDMs treated with or without ketanserin were evaluated by western blot analysis. As demonstrated in Fig. 6E and F, ketanserin markedly decreased the protein level of p-p65 compared with the BMDMs not treated with ketanserin. These data indicate that $5-\mathrm{HT}_{2 \mathrm{~A}} \mathrm{R} /$ $\mathrm{NF}-\kappa \mathrm{B}$ may play a role in the anti-inflammatory effects of ketanserin in macrophages.

\section{Discussion}

The unresolved inflammatory state in the intestinal tract, which was caused by failing to switch from a pro-inflammatory response to an inflammation-resolving anti-inflammatory response, is a characteristic of IBD (29). Both macrophages and DCs actively promote the transition from an inflammatory 

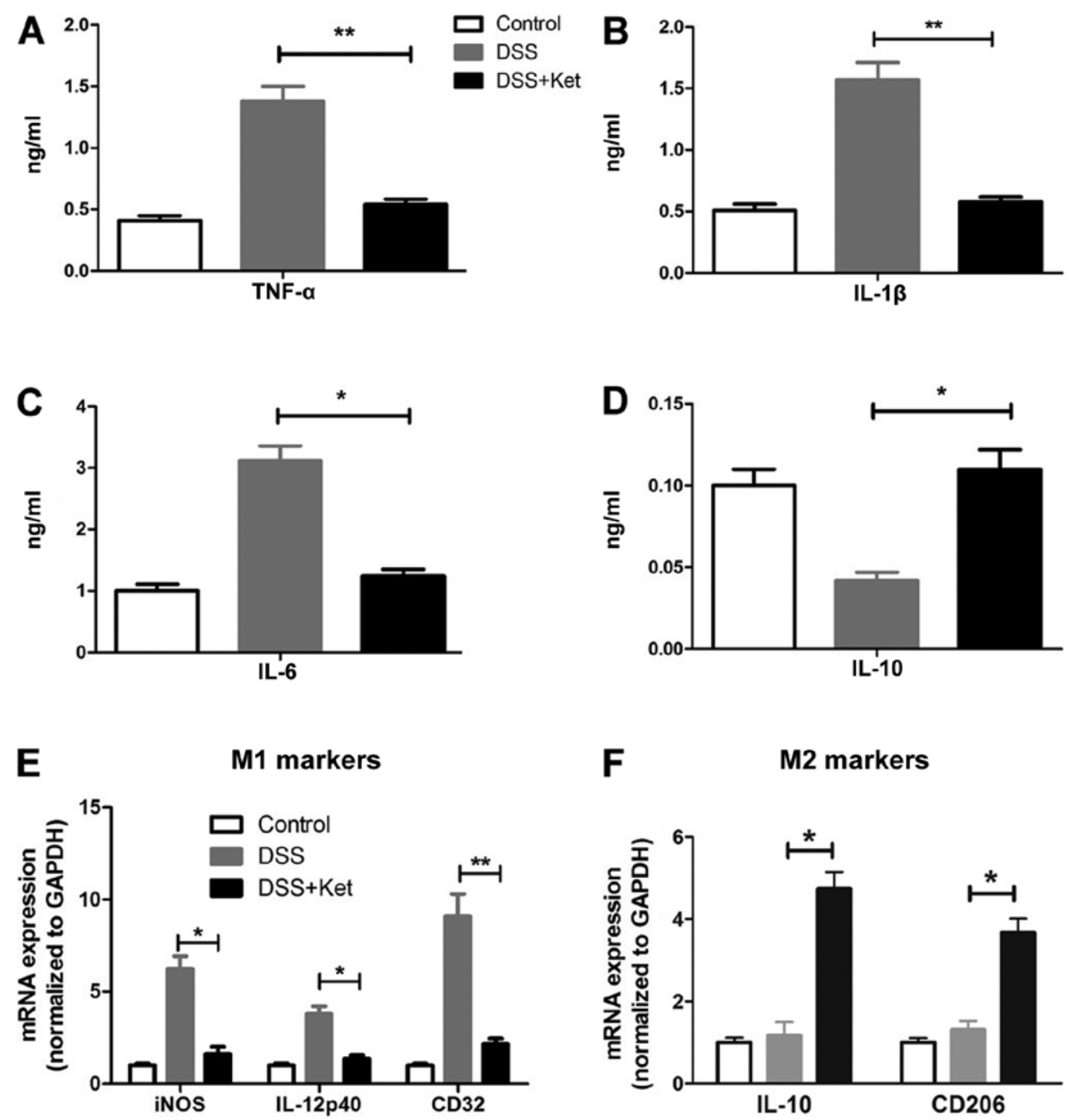

Figure 5. Ketanserin inhibits pro-inflammatory cytokines production and controls the polarization of macrophages. LP-macrophages were obtained from mice in all the 3 groups and stimulated with LPS for $4 \mathrm{~h}$. The production of (A) tumor necrosis factor- $\alpha$ (TNF- $\alpha$ ), (B) interleukin (IL)-1 $1 \beta$, (C) IL-6 and (D) IL-10 was evaluated in the supernatants of LP-macrophage cultures by enzyme-linked immunosorbent assay (ELISA). (E) The mRNA level of M1 polarization markers (iNOS, IL-12 p40 and CD32) and (F) M2 polarization markers (IL-10 and CD206) was evaluated by RT-qPCR. The graphs depict the means \pm SD of 3 independent experiments. $\mathrm{p}<0.05$ and ${ }^{* *} \mathrm{p}<0.01$. Ket, ketanserin.

state to a homeostatic state following immune system activation, and unresolved inflammation is associated with IBD (30). Macrophages are a highly heterogeneous population of cells with a continuum of activation states (31). Evidence indicates that of the macrophages in the lamina propria, the pro-inflammatory $\mathrm{M} 1$ subtype is more prominent in patients with $\mathrm{CD}$ and less prominent in patients with UC (32).

In the present study, we found that $5-\mathrm{HT}_{2 \mathrm{~A}} \mathrm{R}$, which was elevated in colitis, was specifically enhanced in $\mathrm{CD} 68^{+}$ macrophages. Treatment with ketanserin, a selective $5-\mathrm{HT}_{2 \mathrm{~A}} \mathrm{R}$ antagonist, significantly decreased the DSS-induced production of iNOS and pro-inflammatory cytokines (TNF- $\alpha$ and IL-6) in the colons of mice with experimental colitis, with a marked reduction in the infiltration of neutrophils and macrophages in the intestinal mucosa, without a significant difference in the frequency of DCs. Furthermore, ketanserin inhibited the production of iNOS, CD32 and IL-12 in the LP-macrophages upon challenge with LPS, whereas the production of IL-10 and
CD206 increased. This indicates that ketanserin may influence macrophages by promoting their anti-inflammatory function and alterating the polarization from M1 to M2.

The change in the cytokine profile in the ketanserintreated macrophages is consistent with that of other studies. Ahonen et al observed significantly higher levels of IL-1 and IL-6 in LPS-stimulated macrophages following incubation with 5-HT in their study (33). In the study by Liu et al, ketanserin markedly reduced the expression of iNOS, TNF- $\alpha$ and IL-6, as well as that of reactive oxygen species in RAW264.7 cells upon challenge with LPS (12).

The murine chemokines, $\mathrm{KC}$ and $\mathrm{CXCL} 12$, are required for neutrophil and macrophage recruitment, respectively to the sites of inflammation. Our results indicated that ketanserin induced a reduction in the $\mathrm{KC}$ level along with a considerable decrease in the number of macrophages in the colonic mucosa, suggesting that the efficacy of ketanserin may be related to a lower neutrophil and macrophage influx to the site of injury. 

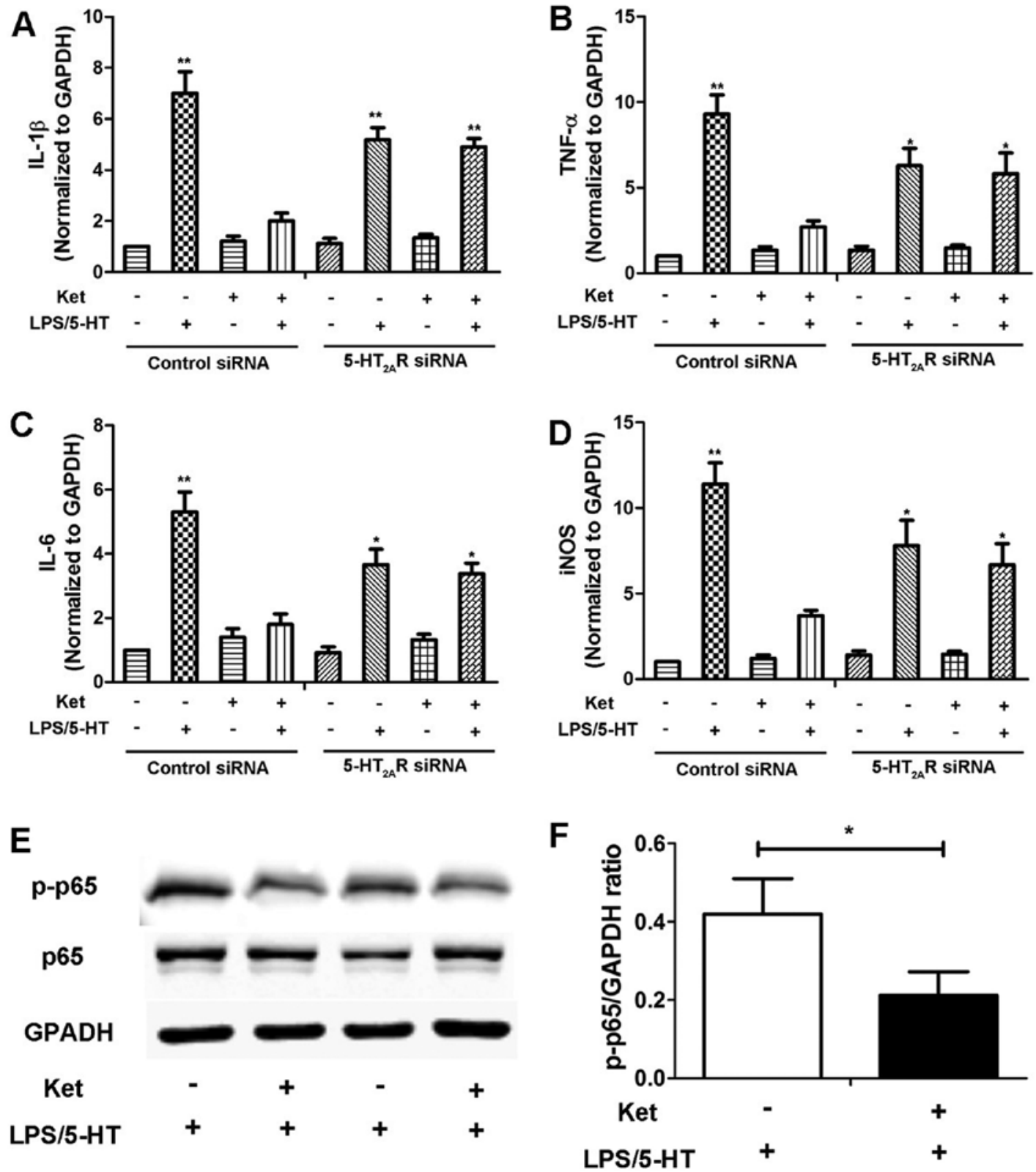

Figure 6. Ketanserin inhibits the expression of pro-inflammatory cytokines and influences the polarization of macrophages partly through 5-hydroxytryptamine-2A receptor $\left(5-\mathrm{HT}_{2 \mathrm{~A}} \mathrm{R}\right)$ /nuclear factor- $\mathrm{kB}(\mathrm{NF}-\mathrm{\kappa B})$. Bone marrow-derived macrophages (BMDMs) were transfected with control siRNA or 5-HT ${ }_{2 \mathrm{~A}} \mathrm{R}$ siRNA followed by stimulation with LPS plus 5-HT (LPS/5-HT). Relative mRNA level expression of (A) interleukin (IL)-1 $\beta$, (B) necrosis factor- $\alpha$ (TNF- $\alpha$ ), (C) IL-6 and (D) iNOS was evaluated in BMDMs by RT-qPCR. (E) Changes in pNF-кB p65 levels in LPS/5-HT-treated BMDMs were evaluated by western blot analyses. (F) Protein levels of pNF-kB p65 were quantified by densitometric analysis. The graphs depict the means \pm SD of 3 independent experiments. ${ }^{*} \mathrm{p}<0.05$ and ${ }^{* * *} \mathrm{p}<0.01$ vs. LPS/5HT with ket-treated BMDMs.

In a previous study, there was also a significant decrease in macrophage infiltration in $\mathrm{TPH} 1^{-/-}$mice with DSS-induced colitis (5). In addition, we demonstrated that ketanserin plays a direct inhibit role in the migration of macrophages, without affecting macrophage phagocytosis.

We also examined 5- $\mathrm{HT}_{2 \mathrm{~A}} \mathrm{R}$ expression in colitis and found that macrophages expressed high levels of $5-\mathrm{HT}_{2 \mathrm{~A}} \mathrm{R}$ in the inflamed colon. 5- $\mathrm{HT}_{2 \mathrm{~A}} \mathrm{R}$ expression has been also shown to be increased in other inflammatory conditions, such as allergic contact dermatitis (34). Furthermore, we demonstrated that $5-\mathrm{HT}_{2 \mathrm{~A}} \mathrm{R}$ siRNA partly reversed the anti-inflammatory effects of ketanserin in BMDMs, thus demonstrating that the inhibitory effects of ketanserin are partly-dependent on 5-HT2AR.
In addition, we demonstrated that ketanserin inhibits NF- $\kappa \mathrm{B}$ activation in BMDMs. These findings reveal an essential role of $5-\mathrm{HT}_{2 \mathrm{~A}} \mathrm{R} / \mathrm{NF}-\kappa \mathrm{B}$ in the effects of ketanserin on the release of cytokines.

In conclusion, the results presented herein demonstrate that treatment with ketanserin attenuated the progression of DSS-induced colitis in mice. The mechanisms responsible for these effects include the inhibition of the release of inflammatory cytokines and the inhibition of the migration of macrophages, but not of their phagocytic ability. Ketanserin also promoted the M2 polarization of colonic macrophages. Moreover, $5-\mathrm{HT}_{2 \mathrm{~A}} \mathrm{R} / \mathrm{NF}-\mathrm{\kappa B}$ may play a role in the therapeutic effects of ketanserin in colitis. 


\section{References}

1. Ravi A, Garg P and Sitaraman SV: Matrix metalloproteinases in inflammatory bowel disease: Boon or a bane? Inflamm Bowel Dis 13: 97-107, 2007.

2. Linden DR, Chen JX, Gershon MD, Sharkey KA and Mawe GM: Serotonin availability is increased in mucosa of guinea pigs with TNBS-induced colitis. Am J Physiol-Gastr L 285: G207-G216, 2003.

3. Oshima S, Fujimura $M$ and Fujimiya $M$ : Changes in number of serotonin-containing cells and serotonin levels in the intestinal mucosa of rats with colitis induced by dextran sodium sulfate. Histochem Cell Biol 112: 257-263, 1999.

4. Bishop AE, Pietroletti R, Taat CW, Brummelkamp WH and Polak JM: Increased populations of endocrine cells in Crohn's ileitis. Virchows Arch A Pathol Anat Histopathol 410: 391-396, 1987.

5. Ghia JE, Li N, Wang HQ, Collins M, Deng YK, El-Sharkawy RT, Cote F, Mallet J and Khan WI: Serotonin has a key role in pathogenesis of experimental colitis. Gastroenterology 137: 1649-1660, 2009 .

6. Coates MD, Mahoney CR, Linden DR, Sampson JE, Chen J, Blaszyk H, Crowell MD, Sharkey KA, Gershon MD and Mawe GM: Molecular defects in mucosal serotonin content and decreased serotonin reuptake transporter in ulcerative colitis and irritable bowel syndrome. Gastroenterology 126: 1657-1664, 2004.

7. Bischoff SC, Mailer R, Pabst O, Weier G, Sedlik W, Li Z, Chen JJ, Murphy DL and Gershon MD: Role of serotonin in intestinal inflammation: Knockout of serotonin reuptake transporter exacerbates 2,4,6-trinitrobenzene sulfonic acid colitis in mice. Am J Physiol Gastrointest Liver Physiol 296: G685-G695, 2009.

8. Regmi SC, Park SY, Ku SK and Kim JA: Serotonin regulates innate immune responses of colon epithelial cells through Nox2-derived reactive oxygen species. Free Radic Biol Med 69: 377-389, 2014.

9. Wang D, Chen T, Gao Y, Quirion R and Hong Y: Inhibition of SNL-induced upregulation of CGRP and NPY in the spinal cord and dorsal root ganglia by the 5-HT(2A) receptor antagonist ketanserin in rats. Pharmacol Biochem Behav 101: 379-386, 2012.

10. Ito $T$, Ikeda U, Shimpo M, Yamamoto $\mathrm{K}$ and Shimada K: Serotonin increases interleukin-6 synthesis in human vascular smooth muscle cells. Circulation 102: 2522-2527, 2000.

11. Lau WK, Chan SC, Law AC, Ip MS and Mak JC: The role of MAPK and Nrf2 pathways in ketanserin-elicited attenuation of cigarette smoke-induced IL-8 production in human bronchial epithelial cells. Toxicol Sci 125: 569-577, 2012.

12. Liu C, Zhang X, Zhou JX, Wei W, Liu DH, Ke P, Zhang GF, Cai GJ and $\mathrm{Su}$ DF: The protective action of ketanserin against lipopolysaccharide-induced shock in mice is mediated by inhibiting inducible NO synthase expression via the MEK/ERK pathway. Free Radic Biol Med 65: 658-666, 2013.

13. Liu C, Zhang GF, Song SW, Cai GJ, Liu WH, Miao CY and Su DF: Effects of ketanserin on endotoxic shock and baroreflex function in rodents. J Infect Dis 204: 1605-1612, 2011.

14. Martinez FO, Sica A, Mantovani A and Locati M: Macrophage activation and polarization. Front Biosci 13: 453-461, 2008.

15. Mosser DM and Edwards JP: Exploring the full spectrum of macrophage activation. Nat Rev Immunol 8: 958-969, 2008.

16. Bettelli E, Carrier Y, Gao W, Korn T, Strom TB, Oukka M, Weiner HL and Kuchroo VK: Reciprocal developmental pathways for the generation of pathogenic effector TH17 and regulatory T cells. Nature 441: 235-238, 2006.
17. Xie N, Cui H, Banerjee S, Tan Z, Salomao R, Fu M, Abraham E, Thannickal VJ and Liu G: miR-27a regulates inflammatory response of macrophages by targeting IL-10. J Immunol 193: 327-334, 2014

18. Hernandez ME,Martinez-Fong D,Perez-Tapia M,Estrada-Garcia I, Estrada-Parra S and Pavon L: Evaluation of the effect of selective serotonin-reuptake inhibitors on lymphocyte subsets in patients with a major depressive disorder. Eur Neuropsychopharmacol 20: 88-95, 2010.

19. Young MR and Matthews JP: Serotonin regulation of T-cell subpopulations and of macrophage accessory function. Immunology 84: 148-152, 1995.

20. Berndt BE, Zhang M, Chen GH, Huffnagle GB and Kao JY: The role of dendritic cells in the development of acute dextran sulfate sodium colitis. J Immunol 179: 6255-6262, 2007.

21. Kitajima S, Takuma S and Morimoto M: Changes in colonic mucosal permeability in mouse colitis induced with dextran sulfate sodium. Exp Anim 48: 137-143, 1999.

22. Hunter MM, Wang A, Parhar KS, Johnston MJ, Van Rooijen N, Beck PL and McKay DM: In vitro-derived alternatively activated macrophages reduce colonic inflammation in mice. Gastroenterology 138: 1395-1405, 2010.

23. Qualls JE, Kaplan AM, van Rooijen N and Cohen DA: Suppression of experimental colitis by intestinal mononuclear phagocytes. J Leukoc Biol 80: 802-815, 2006.

24. Beider K, Bitner H, Leiba M, Gutwein O, Koren-Michowitz M, Ostrovsky O, Abraham M, Wald H, Galun E, Peled A and Nagler A: Multiple myeloma cells recruit tumor-supportive macrophages through the CXCR4/CXCL12 axis and promote their polarization toward the M2 phenotype. Oncotarget 5: 11283-11296, 2014.

25. Kim D, Kim J, Yoon JH, Ghim J, Yea K, Song P, Park S, Lee A, Hong CP, Jang MS, et al: CXCL12 secreted from adipose tissue recruits macrophages and induces insulin resistance in mice. Diabetologia 57: 1456-1465, 2014.

26. Maneglier B, Guillemin G, Clayette P, Rogez-Kreuz C, Brew B, Dormont D, Advenier C, Therond $\mathrm{P}$ and Spreux-Varoquaux O: Serotonin decreases HIV-1 replication in primary cultures of human macrophages through $5-\mathrm{HT}(1 \mathrm{~A})$ receptors. Br J Pharmacol 154: 174-182, 2008.

27. Hayden MS, West AP and Ghosh S: NF-kappaB and the immune response. Oncogene 25: 6758-6780, 2006.

28. Atreya I, Atreya R and Neurath MF: NF-kappaB in inflammatory bowel disease. J Intern Med 263: 591-596, 2008.

29. Steinbach EC and Plevy SE: The role of macrophages and dendritic cells in the initiation of inflammation in IBD. Inflamm Bowel Dis 20: 166-175, 2014.

30. Nathan C and Ding AH: Nonresolving Inflammation. Cell 140: 871-882, 2010.

31. Murray PJ and Wynn TA: Protective and pathogenic functions of macrophage subsets. Nat Rev Immunol 11: 723-737, 2011.

32. Smith AM, Rahman FZ, Hayee B, Graham SJ, Marks DJ, Sewell GW, Palmer CD, Wilde J, Foxwell BM, Gloger IS, et al: Disordered macrophage cytokine secretion underlies impaired acute inflammation and bacterial clearance in Crohn's disease. J Exp Med 206: 1883-1897, 2009.

33. Ahonen A, Kyosola K and Penttila O: Enterochromaffin cells in macrophages in ulcerative colitis and irritable colon. Ann Clin Res 8: 1-7, 1976.

34. Wetterberg J, Taher C, Azmitia EC and El-Nour H: Timedependent modulation of serotonin and its receptors $1 \mathrm{~A}$ and $2 \mathrm{~A}$ expression in allergic contact dermatitis. J Eur Acad Dermatol 25: 1200-1205, 2011. 